\title{
Mono-substituted molybdenium preyssler heteropolyacid: An ecofriendly photocatalyst for the syntheses of gold nanoparticles in solution and titanium dioxide surface with excellent photoactivity in combination with titanium dioxide
}

\author{
Majid M. Heravi ${ }^{1}$, Fatemeh F. Bamoharram, Saideh Kadkhodaei, Ali Ayati, Javad Bahara \\ ${ }^{1}$ Department of Chemistry, Faculty of Physics \&Chemistry Alzahra University, Vanak, Tehran, Iran.
}

\begin{abstract}
Gold nanoparticles were synthesized, using a straightforward photoreduction protocol in the presence of mixedaddenda Preyssler heteropolyacid, $\mathrm{H} 14[\mathrm{NaP5W} 29 \mathrm{MoO} 110]$, (PMo). It acts as a reductive agent, as well as a photocatalyst and stabilizing agent. The obtained results showed that the construction rate, morphology and the size of the gold nanoparticles strongly depend on the volume of propan-2-ol amount, used as solvent in the reaction. The shapes of the synthesized gold nanoparticles in the low volume of the solvent were almost uniform spheres with the size of $40-60 \mathrm{~nm}$. The morphologies of the gold nanoparticles in higher volume of the same solvent were found to involve a mixture of triangular, tubular, pentagonal and hexagonal structures along with spheres in range of 5-30 nm. Besides, the ability of the PMo as reductive agent for the syntheses of gold nanoparticles onto the surface of TiO2via photochemical technique was investigated. The results showed that, PMo as a reducing catalytic linker could be bound onto titanium dioxide for syntheses of the desired gold nanoparticles. The synthesized gold nanoparticles and nanocomposites were characterized by TEM, XRD, SEM, UV and FTIR techniques. The photocatalytic activity of the synthesized nanocomposite was measured by photodegradation of the organic dye "malachite green" under UV-Vis light and was found to be excellent.
\end{abstract}

\section{Keyword}

Gold nanoparticles, Heteropolyacid, Mixed-addenda Preyssler, Nanocomposite, Photocatalyst, Titanium dioxide, Fiber optic sensors, Metal nanoparticles, Molybdenum compounds, Morphology, Nanocomposites, Oxides. 